Case Report

\title{
Bone and Joint Infections due to Haemophilus parainfluenzae: Case Report and Review of the Literature
}

\author{
Conar R. O'Neil, ${ }^{1}$ Evan Wilson, ${ }^{2}$ and Bayan Missaghi ${ }^{2}$ \\ ${ }^{1}$ Department of Internal Medicine, Cumming School of Medicine, Health Sciences Centre, University of Calgary, Foothills Campus, \\ 3330 Hospital Drive NW, Calgary, AB, Canada T2N 4N1 \\ ${ }^{2}$ Department of Microbiology, Immunology and Infectious Disease, Cumming School of Medicine, Health Sciences Centre, \\ University of Calgary, Foothills Campus, 3330 Hospital Drive NW, Calgary, AB, Canada T2N 4N1
}

Correspondence should be addressed to Bayan Missaghi; bayan.missaghi@albertahealthservices.ca

Received 2 July 2015; Accepted 15 December 2015

Copyright (c) 2016 Conar R. O’Neil et al. This is an open access article distributed under the Creative Commons Attribution License, which permits unrestricted use, distribution, and reproduction in any medium, provided the original work is properly cited.

Haemophilus parainfluenzae is a normal inhabitant of the human respiratory tract. However it is an increasingly recognized pathogen in invasive infections, particularly in the immunocompromised host and where there is disruption of the normal skin or mucosal barriers. We present a case of a 56-year-old female with a history of asplenia who developed H. parainfluenzae septic arthritis of the hip following an intra-articular steroid injection. We also summarize previously reported cases of bone and joint infections caused by $H$. parainfluenzae.

\section{Case Presentation}

A 56-year-old female presented to the emergency room with a three-day history of right hip pain. She reported decreased range of motion and difficulty ambulating. She denied constitutional symptoms or fever. She had undergone intra-articular steroid injection of her right hip three days prior. Review of systems was otherwise unremarkable. Her past medical history was relevant for hereditary spherocytosis with splenectomy at age 14 and chronic right hip osteoarthritis. Her only medication was celecoxib as needed. She denied any drug allergy. She was unaware of her immunization history. She worked as a flight attendant, denied smoking or recreational drug use, and had not recently travelled outside of Canada. On initial examination she was afebrile and her vital signs were within normal limits. On examination of her right hip she had limitation of internal rotation with reproducible pain, but her physical exam otherwise was unremarkable. Her initial investigations showed elevation in C-reactive protein (CRP) and erythrocyte sedimentation rate (ESR) at $22.9 \mathrm{mg} / \mathrm{L}$ and $25 \mathrm{~mm} / \mathrm{hr}$, respectively. Her white blood cell count was not elevated. An X-ray of her right hip showed severe joint space narrowing with osteophytosis consistent with severe osteoarthritis. Arthrocentesis was performed and synovial fluid samples were directly inoculated onto solid culture media, including blood agar and chocolate agar. Two of three samples also underwent cytospin centrifugation and direct Gram stain. Gram-negative bacilli were observed in one sample though heavy neutrophils were observed in both. Synovial fluid analysis for cell count and chemistry was not performed due to insufficient sample. She was admitted to hospital for 24 hours of observation and was subsequently discharged with instructions to return to hospital if her symptoms worsened or if cultures subsequently grew a pathogenic organism.

Three days later she was seen in a follow-up clinic. In the interim she had developed a fever $\left(38.3^{\circ} \mathrm{C}\right)$, had chills, and had worsening right hip pain. Repeat physical examination showed deterioration in range of motion at her right hip with significant pain. Cardiovascular examination was unremarkable with no appreciable murmur. Synovial fluid cultures from the initial arthrocentesis were now growing Haemophilus parainfluenzae with colonies observed on solid media within 24 hours of inoculation. She received one dose of ceftriaxone and subsequently underwent right hip arthrotomy with synovectomy and irrigation. The Infectious Disease service was consulted the following day. The organism was susceptible to ceftriaxone and cefuroxime and resistant to ampicillin and ciprofloxacin (Table 1). Blood cultures and intraoperative tissue cultures did not demonstrate growth, 
TABLE 1: Haemophilus parainfluenzae susceptibility results.

\begin{tabular}{|c|c|c|}
\hline Antibiotic & Result & Method \\
\hline Beta-lactamase & Positive & $\begin{array}{l}\text { Nitrocefin SR112, Oxoid } \\
\text { Microbiology Products }\end{array}$ \\
\hline Ciprofloxacin & Resistant & $\begin{array}{c}\text { MIC by Etest }=6 \mathrm{ug} / \mathrm{mL} \\
\text { Sensitive } \leq 1\end{array}$ \\
\hline Ceftriaxone & Susceptible (32 mm) & Kirby Bauer \\
\hline Cefuroxime & Susceptible (30 mm) & Kirby Bauer \\
\hline Meropenem & Susceptible $(28 \mathrm{~mm})$ & Kirby Bauer \\
\hline
\end{tabular}

Disk diffusion and Etest as per CLSI M100 S24 document; Haemophilus test medium: $35^{\circ} \mathrm{C} \pm 2^{\circ} \mathrm{C}$ in $5 \% \mathrm{CO}_{2}$ for $16-18$ hours.

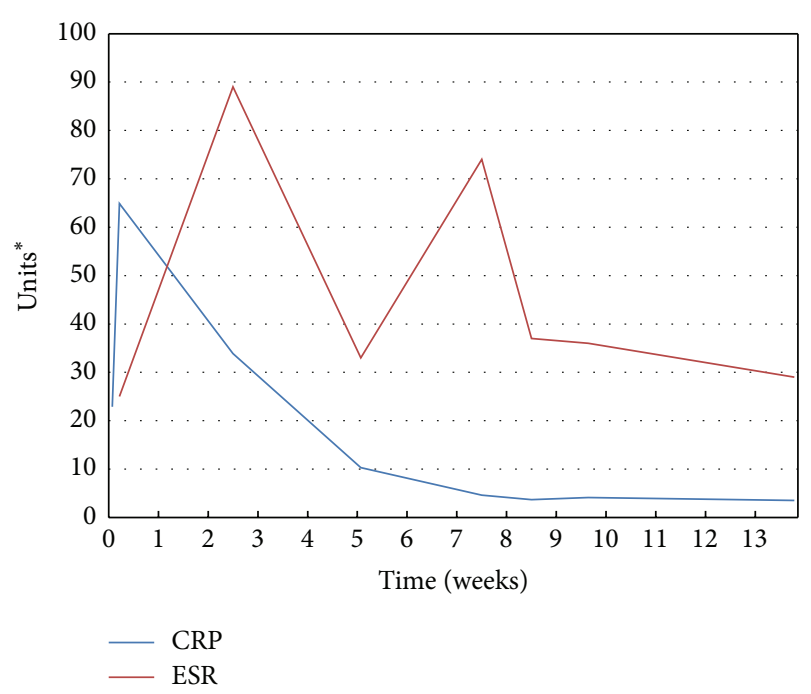

FIGURE 1: Inflammatory markers over time during a prolonged course of intravenous ceftriaxone for Haemophilus parainfluenzae septic arthritis. CRP: C-reactive protein; ESR: erythrocyte sedimentation rate. ${ }^{*} \mathrm{CRP}=\mathrm{mg} / \mathrm{L} ; \mathrm{ESR}=\mathrm{mm} / \mathrm{hr}$.

likely due to the administration of antibiotics prior to collection. The patient was treated with intravenous ceftriaxone and was subsequently discharged with outpatient follow-up for home intravenous antibiotics. Ongoing pain, difficulty ambulating, and persistently elevated inflammatory markers necessitated a prolonged, nine-week course of antimicrobial therapy (Figure 1). At the time of treatment discontinuation, she reported pain and her functional ability had not yet returned to baseline. She is currently awaiting evaluation for total hip arthroplasty.

\section{Discussion}

$H$. parainfluenzae is a pleomorphic Gram-negative coccobacillus with fastidious growth requirements, which require enriched media, usually containing blood (e.g., chocolate agar). It can be differentiated from other Haemophilus spp. by the requirement for $\mathrm{V}$ factor (i.e., NAD, nicotinamide adenine dinucleotide) for growth [1]. H. parainfluenzae is part of the normal flora of the oral cavity and respiratory tract [1]. It is an increasingly recognized opportunistic pathogen in serious infections such as endocarditis, meningitis, and pneumonia and has also been recognized as a rare cause of nongonococcal urethritis [2]. However, it is an uncommon pathogen in osteomyelitis and septic arthritis.

Inclusive of our case, there have been only 16 cases of bone and joint infections caused by $H$. parainfluenzae reported in the English literature (summarized in Table 2) [3-16]. Of these 16 patients, 10 (63\%) had septic arthritis, four (25\%) had osteomyelitis, and two (13\%) had both septic arthritis and osteomyelitis. The median age was 65 (Interquartile Range [IQR]: 46-76) and 63\% were male. Most (14/16, 88\%) were immunocompetent and only one reported recent trauma [14]. Four patients (25\%) had prosthetic joint infections. Most patients $(10 / 16,63 \%)$ reported a procedure in the previous three months (four dental, two nasopharyngeal, two gastrointestinal, one total knee arthroplasty, and one intraarticular steroid injection). The organism was identified on culture of aspirated synovial fluid (6/16, 38\%), blood (4/16, $25 \%)$, and surgical or biopsy specimens (7/16, 44\%). 8/16 $(50 \%)$ of patients required surgical intervention and the rest $(8 / 16,50 \%)$ were managed with antibiotics alone. One patient with a prosthetic joint infection was treated with chronic, suppressive antibiotic therapy after having refused surgical intervention [10]. The median length of antibiotic therapy was 42 days (IQR: 14-70). Of patients with data reported, most $(11 / 14,79 \%)$ were treated with a beta-lactam antibiotic, the most common being ampicillin (7/14, 50\%).

To our knowledge, we report the first case of septic arthritis due to a beta-lactamase producing strain of $H$. parainfluenzae (Table 1). Community surveillance studies of Haemophilus spp. isolated from respiratory samples have reported rates of beta-lactamase production as high as $70 \%$ $[17,18]$. Beta-lactamase plasmids can transmit from less pathogenic strains of Haemophilus, such as H. parainfluenzae, to invasive pathogens such as Haemophilus influenza type b, suggesting $H$. parainfluenzae may be a reservoir for antimicrobial resistance in the nasopharyngeal tract [18]. More recently, increasing rates of antimicrobial resistance have also been reported in genitourinary isolates of $H$. parainfluenzae $[2,19]$.

Moreover, it is well known that immunocompromised hosts are particularly susceptible to opportunistic pathogens, and it is within this context that we report the first case of septic arthritis due to $H$. parainfluenzae in a patient with asplenia. Patients with asplenia are at significant increased risk of sepsis due to encapsulated bacteria, such as Haemophilus spp., Neisseria spp., and Streptococcus pneumoniae [20]. Immunizations for pneumococcal disease, Haemophilus influenzae type $b$ infection, and meningococcal disease are recommended for asplenic patients. However, they remain susceptible to less common potentially pathogenic bacteria, such as $H$. parainfluenzae. Prompt recognition and source control (e.g., surgical debridement) are particularly important in preventing disseminated infection in patients with asplenia. In our case, given her risk of disseminated infection, surgical intervention and antibiotics could have been initiated sooner, when Gram stain of the initial arthrocentesis was reported. 


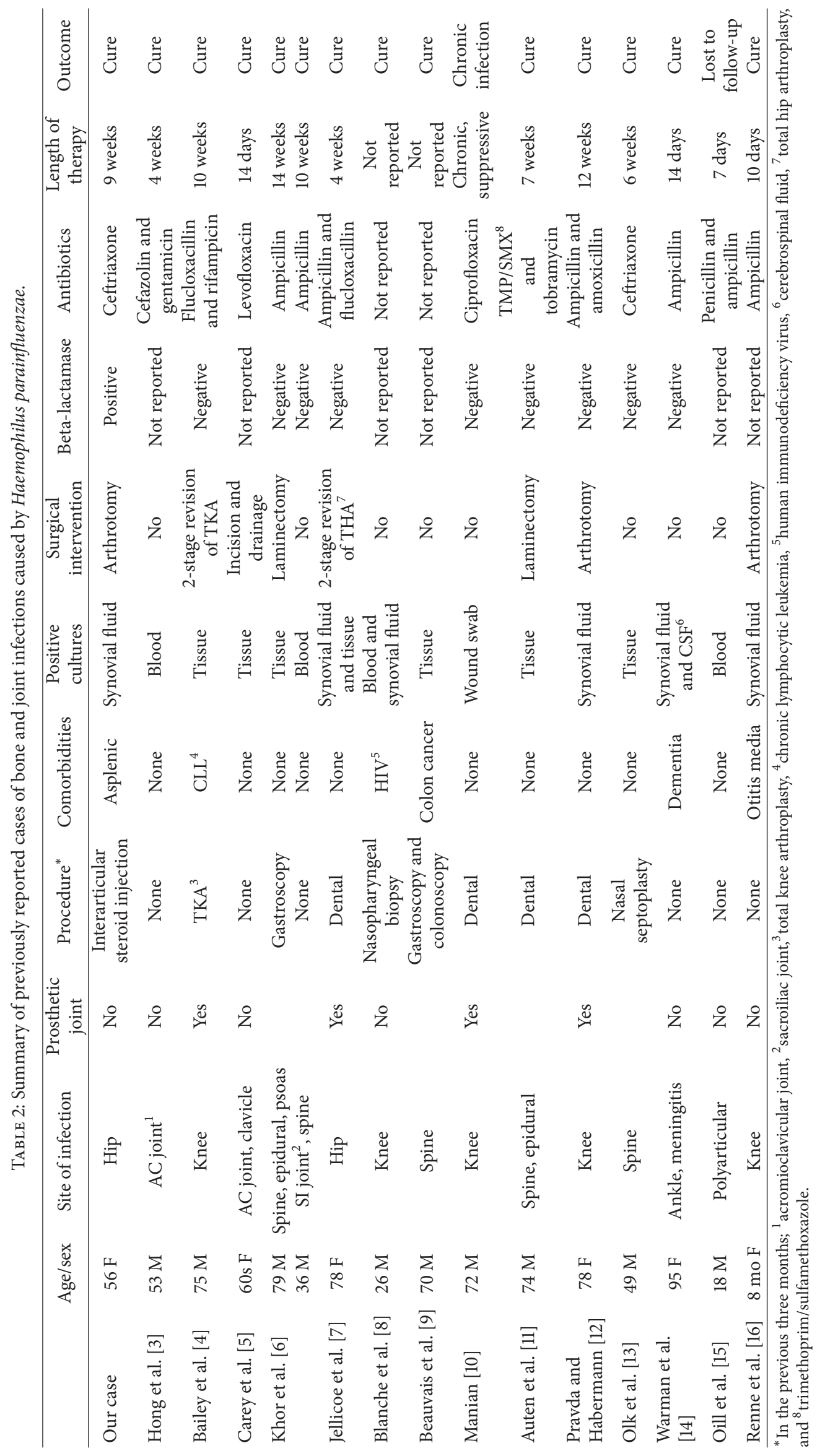


To the best of our knowledge, our case is the only example of $H$. parainfluenzae septic arthritis temporally associated with intra-articular steroid injection that is described in the literature. Septic arthritis is a rare but recognized complication following intra-articular steroid injection. Infection may result from lack of adherence to proper aseptic technique during the procedure and subsequent inoculation of the joint space, or through microbiological contamination of the steroid preparation used [21,22]. Alternatively, transient bacteremia can lead to secondary seeding of a susceptible joint capsule, for example, in patients with underlying osteoarthritis or previous joint infection. However, localized immunosuppression from intra-articular steroid injections does not appear to be associated with increased rates of deep infection as demonstrated in patients receiving steroid injection prior to joint arthroplasty $[23,24]$.

$H$. parainfluenzae is an uncommon though increasingly recognized pathogen in bone and joint infections. In this series, patients with prosthetic joints and patients undergoing invasive procedures appear to be particularly susceptible to this pathogen. Furthermore, the rate of beta-lactamase producing strains of $H$. parainfluenzae may be increasing. Thus, accurate microbiologic diagnosis is key to providing tailored antibiotics, as patients often require a prolonged course of therapy.

\section{Competing Interests}

The authors have no conflict of interests regarding this paper.

\section{Acknowledgments}

The authors thank Dr. Julie Carson.

\section{References}

[1] G. F. Brooks, K. C. Carroll, J. S. Butel, S. A. Morse, and T. A. Mietzner, "Haemophilus, Bordetella, Brucella, and Francisella," in Jawetz, Melnick, \& Adelberg's Medical Microbiology, chapter 18, McGraw-Hill, New York, NY, USA, 26th edition, 2013.

[2] G. Deza, G. Martin-Ezquerra, J. Gómez, J. Villar-García, A. Supervia, and R. M. Pujol, "Isolation of Haemophilus influenzae and Haemophilus parainfluenzae in urethral exudates from men with acute urethritis: a descriptive study of 52 cases," Sexually Transmitted Infections, vol. 92, no. 1, pp. 29-31, 2016.

[3] M. J. Hong, Y. D. Kim, and H. D. Ham, "Acute septic arthritis of the acromioclavicular joint caused by Haemophilus parainfluenzae: a rare causative origin," Clinical Rheumatology, vol. 34, no. 4, pp. 811-814, 2014.

[4] C. Bailey, S. Duckett, S. Davies, R. Townsend, and I. Stockley, "Haemophilus parainfluenzae prosthetic joint infection. The importance of accurate microbiological diagnosis and options for management," Journal of Infection, vol. 63, no. 6, pp. 474476, 2011.

[5] T. W. Carey, K. Jackson, R. Roure, and B. E. Abell, "Acromioclavicular septic arthritis: a case report of a novel pathogen," The American Journal Of Orthopedics, vol. 39, no. 3, pp. 134-136, 2010.
[6] B.-S. Khor, S.-J. Liaw, H.-C. Liao, C.-C. Yang, and M.-H. Lee, "Bone and joint infections caused by Haemophilus parainfluenzae: report of two cases and literature review," Infectious Diseases in Clinical Practice, vol. 15, no. 3, pp. 206-208, 2007.

[7] P. A. Jellicoe, A. Cohen, and P. Campbell, "Haemophilus parainfluenzae complicating total hip arthroplasty: a rapid failure," Journal of Arthroplasty, vol. 17, no. 1, pp. 114-116, 2002.

[8] P. Blanche, S. Abad, and D. Sicard, "Septic arthritis due to Haemophilus parainfluenzae in a patient infected with the human immunodeficiency virus type 1," Revue du Rhumatisme (English Edition), vol. 63, no. 5, article 380, 1996.

[9] C. Beauvais, F. Berenbaum, M. Spentchian, A. Prier, and G. Kaplan, "Early diagnosis of vertebral osteomyelitis due to a rare pathogen: Haemophilus parainfluenzae," Journal of Rheumatology, vol. 19, no. 3, pp. 491-493, 1992.

[10] F. A. Manian, "Prosthetic joint infection due to Haemophilus parainfluenzae after dental surgery," Southern Medical Journal, vol. 84, no. 6, pp. 807-808, 1991.

[11] G. M. Auten, C. S. Levy, and M. A. Smith, "Haemophilus parainfluenzae as a rare cause of epidural abscess: case report and review," Reviews of Infectious Diseases, vol. 13, no. 4, pp. 609-612, 1991.

[12] J. Pravda and E. Habermann, "Hemophilus parainfluenzae complicating total knee arthroplasty. A case report," Clinical Orthopaedics and Related Research, no. 243, pp. 169-171, 1989.

[13] D. G. Olk, R. J. Hamill, and R. A. Proctor, "Haemophilus parainfluenzae vertebral osteomyelitis," The American Journal of the Medical Sciences, vol. 294, no. 2, pp. 114-116, 1987.

[14] S. T. Warman, E. Reinitz, and R. S. Klein, "Haemophilus parainfluenzae septic arthritis in an adult," The Journal of the American Medical Association, vol. 246, no. 8, pp. 868-869, 1981.

[15] P. A. Oill, A. W. Chow, and L. B. Guze, "Adult bacteremic Haemophilus parainfluenzae infections. Seven reports of cases and a review of the literature," Archives of Internal Medicine, vol. 139, no. 9, pp. 985-988, 1979.

[16] J. W. Renne, H. B. Tanowitz, and J. D. Chulay, "Septic arthritis in an infant due to Clostridium ghoni and Hemophilus parainfluenzae," Pediatrics, vol. 57, no. 4, pp. 573-574, 1976.

[17] D. W. Scheifele, S. J. Fusell, and M. C. Roberts, "Characterization of ampicillin-resistant Haemophilus parainfluenzae," Antimicrobial Agents and Chemotherapy, vol. 21, no. 5, pp. 734-739, 1982.

[18] N. I. Leaves, I. Dimopoulou, I. Hayes et al., "Epidemiological studies of large resistance plasmids in Haemophilus," Journal of Antimicrobial Chemotherapy, vol. 45, no. 5, pp. 599-604, 2000.

[19] M. Giufrè, L. Daprai, M. L. Garlaschi, R. Cardines, E. Torresani, and M. Cerquetti, "Genital carriage of the genus Haemophilus in pregnancy: species distribution and antibiotic susceptibility," Journal of Medical Microbiology, vol. 64, no. 7, pp. 724-730, 2015.

[20] L. G. Rubin and W. Schaffner, "Clinical practice. Care of the asplenic patient," The New England Journal of Medicine, vol. 371, no. 4, pp. 349-356, 2014.

[21] J. M. Ritter, A. Muehlenbachs, D. M. Blau et al., "Exserohilum infections associated with contaminated steroid injections: a clinicopathologic review of 40 cases," American Journal of Pathology, vol. 183, no. 3, pp. 881-892, 2013.

[22] M. R. Wong, P. Del Rosso, L. Heine et al., "An outbreak of Klebsiella pneumoniae and Enterobacter aerogenes bacteremia after interventional pain management procedures, New York City, 2008," Regional Anesthesia and Pain Medicine, vol. 35, no. 6, pp. 496-499, 2010. 
[23] D. Marsland, A. Mumith, and I. W. Barlow, "Systematic review: the safety of intra-articular corticosteroid injection prior to total knee arthroplasty," Knee, vol. 21, no. 1, pp. 6-11, 2014.

[24] C. P. Charalambous, A. D. Prodromidis, and T. A. Kwaees, "Do intra-articular steroid injections increase infection rates in subsequent arthroplasty? A systematic review and metaanalysis of comparative studies," Journal of Arthroplasty, vol. 29, no. 11, pp. 2175-2180, 2014. 


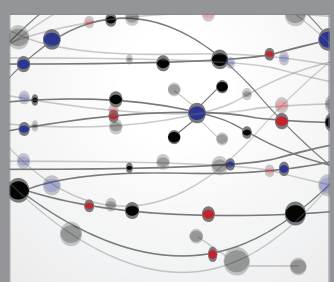

The Scientific World Journal
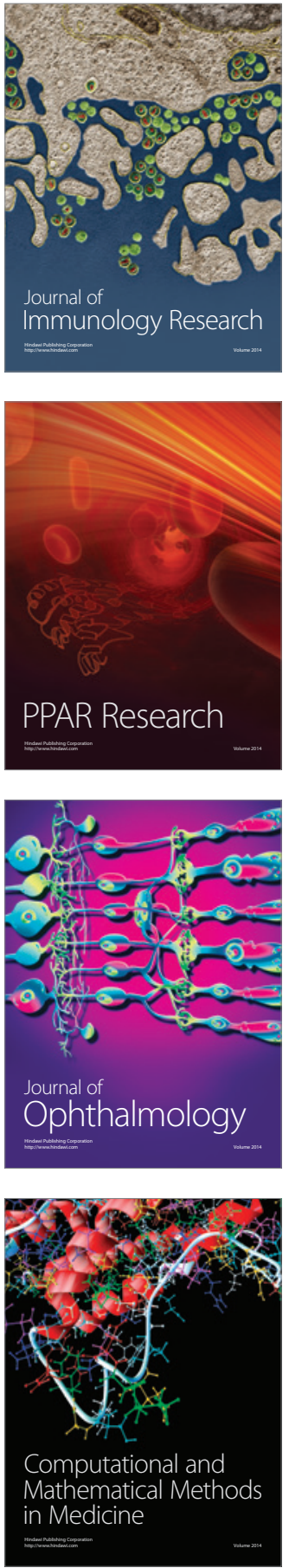

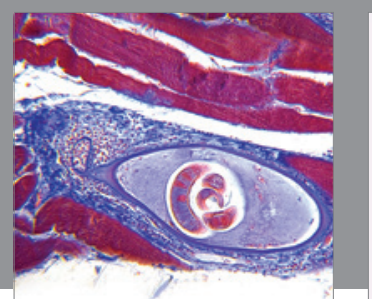

Gastroenterology Research and Practice

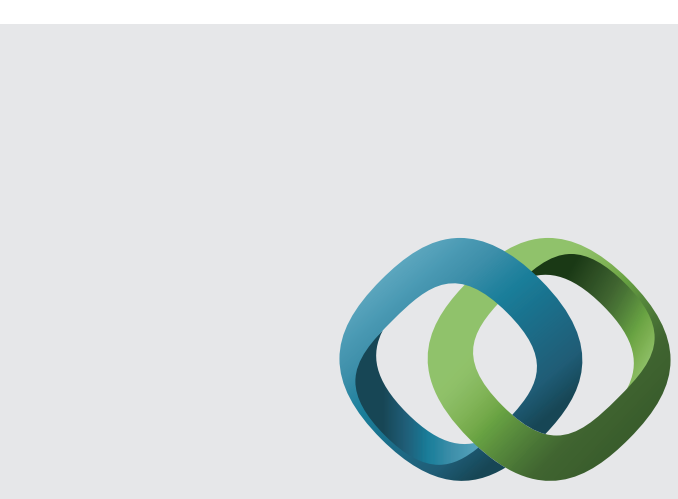

\section{Hindawi}

Submit your manuscripts at

http://www.hindawi.com
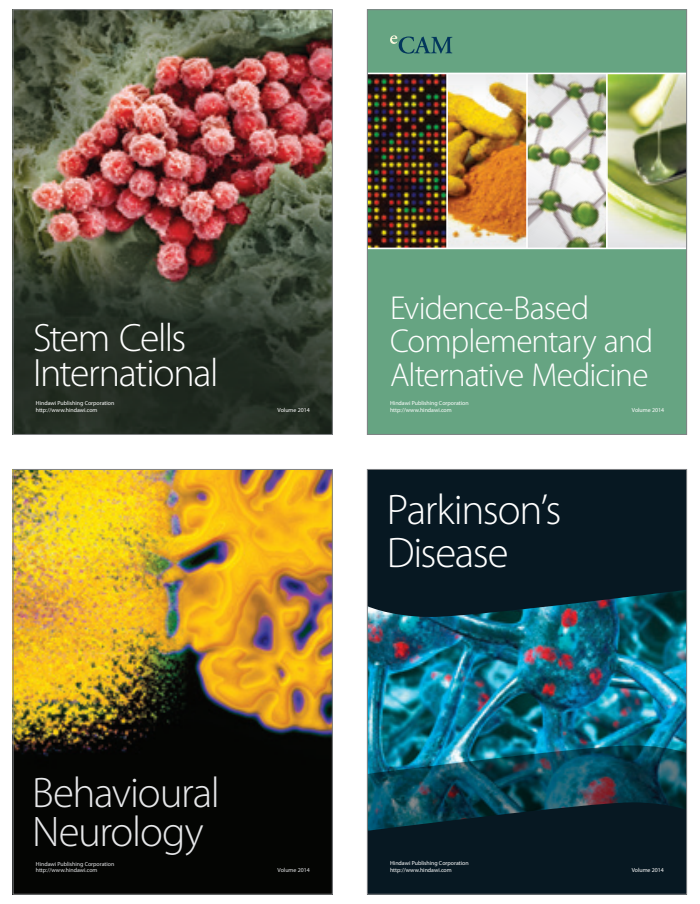
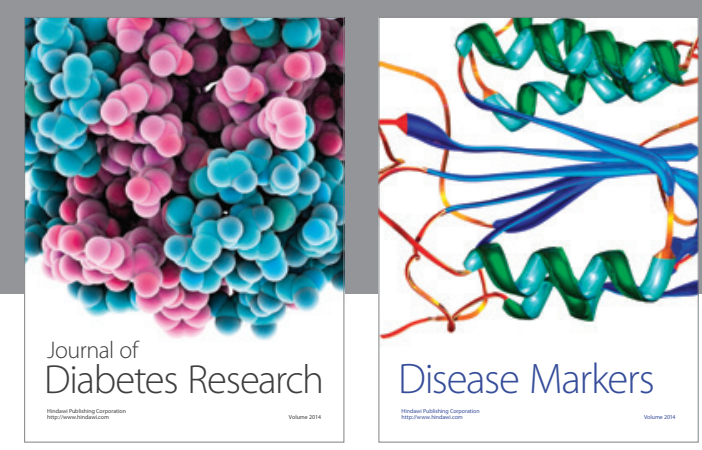

Disease Markers
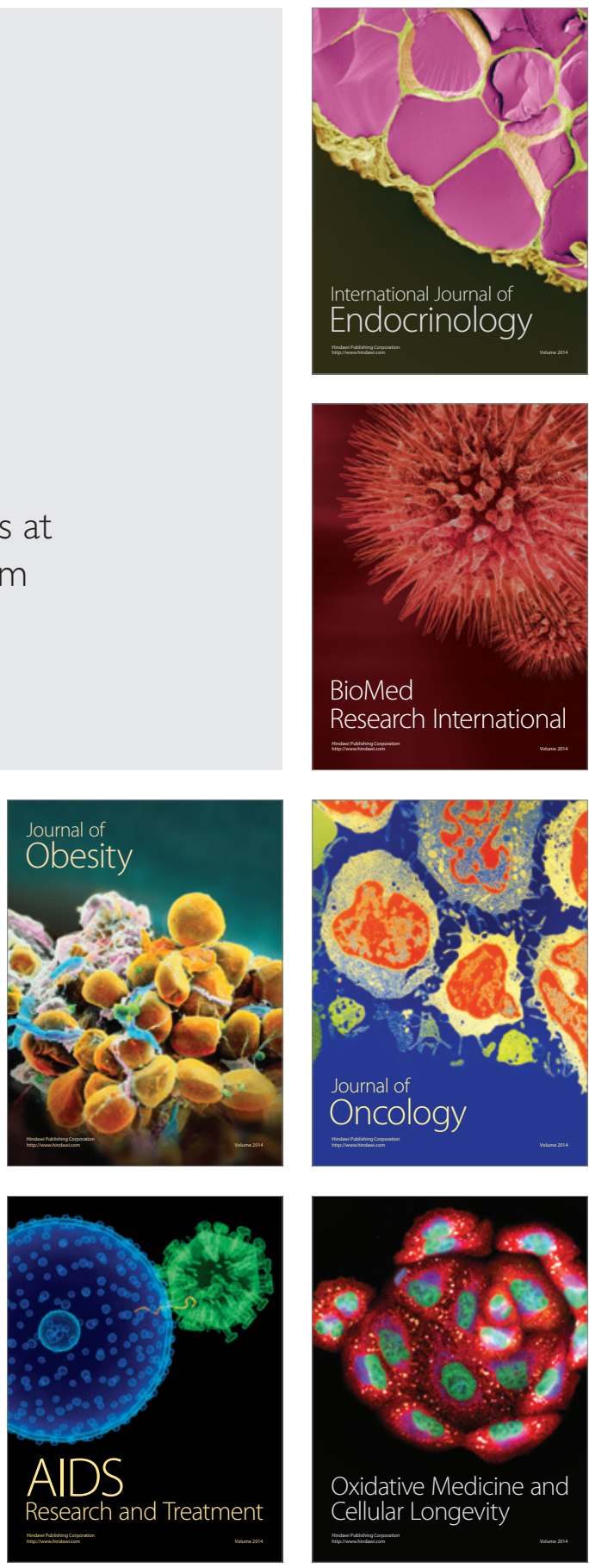